Pacific

Journal of

Mathematics

\title{
FLAG SUBDIVISIONS AND $\boldsymbol{\gamma}$-VECTORS
}

CHRISTOS A. ATHANASIADIS

Volume 259 No. 2

October 2012 


\title{
FLAG SUBDIVISIONS AND $\gamma$-VECTORS
}

\author{
Christos A. ATHANASIADis
}

\begin{abstract}
The $\gamma$-vector is an important enumerative invariant of a flag simplicial homology sphere. It has been conjectured by Gal that this vector is nonnegative for every such sphere $\Delta$ and by Reiner, Postnikov and Williams that it increases when $\Delta$ is replaced by any flag simplicial homology sphere that geometrically subdivides $\Delta$. Using the nonnegativity of the $\gamma$-vector in dimension 3, proved by Davis and Okun, as well as Stanley's theory of simplicial subdivisions and local $\boldsymbol{h}$-vectors, the latter conjecture is confirmed in this paper in dimensions 3 and 4.
\end{abstract}

\section{Introduction}

This paper is concerned with the face enumeration of an important class of simplicial complexes, that of flag homology spheres, and their subdivisions. The face vector of a homology sphere (more generally, of an Eulerian simplicial complex) $\Delta$ can be conveniently encoded by its $\gamma$-vector [Gal 2005], denoted by $\gamma(\Delta)$. Part of our motivation comes from the following two conjectures. (We refer to Section 2 for all relevant definitions.) The first, proposed by Gal [2005, Conjecture 2.1.7], can be thought of as a generalized lower-bound conjecture for flag homology spheres; it strengthens an earlier conjecture by Charney and Davis [1995]. The second, proposed by Postnikov, Reiner and Williams [Postnikov et al. 2008, Conjecture 14.2], is a natural extension of the first.

Conjecture 1.1 [Gal 2005]. For every flag homology sphere $\Delta$ we have $\gamma(\Delta) \geq 0$. Conjecture 1.2 [Postnikov et al. 2008]. For all flag homology spheres $\Delta$ and $\Delta^{\prime}$ for which $\Delta^{\prime}$ geometrically subdivides $\Delta$, we have $\gamma\left(\Delta^{\prime}\right) \geq \gamma(\Delta)$.

These statements are trivial for spheres of dimension 2 or less. Conjecture 1.1 was proved for 3-dimensional spheres by Davis and Okun [2001, Theorem 11.2.1] and was deduced from that result for 4-dimensional spheres in [Gal 2005, Corollary 2.2.3]. Conjecture 1.2 can be thought of as a conjectural analogue of the fact [Stanley 1992, Theorem 4.10] that the $h$-vector (a certain linear transformation of the face

MSC2010: primary 05E45; secondary 05E99.

Keywords: flag complex, homology sphere, simplicial subdivision, flag subdivision, face enumeration, $\gamma$-vector. 
vector) of a Cohen-Macaulay simplicial complex increases under quasigeometric simplicial subdivision (a class of topological subdivisions that includes all geometric simplicial subdivisions). The main result of this paper proves its validity in three and four dimensions for a new class of simplicial subdivisions, which includes all geometric ones.

Theorem 1.3. For every flag homology sphere $\Delta$ of dimension 3 or 4 and for every flag vertex-induced homology subdivision $\Delta^{\prime}$ of $\Delta$, we have $\gamma\left(\Delta^{\prime}\right) \geq \gamma(\Delta)$.

This result naturally suggests the following stronger version of Conjecture 1.2:

Conjecture 1.4. For every flag homology sphere $\Delta$ and every flag vertex-induced homology subdivision $\Delta^{\prime}$ of $\Delta$, we have $\gamma\left(\Delta^{\prime}\right) \geq \gamma(\Delta)$.

The following structural result on flag homology spheres, which may be of independent interest, will also be proved in Section 4. It implies, for instance, that Conjecture 1.4 is stronger than Conjecture 1.1. Throughout this paper, we will denote by $\Sigma_{d-1}$ the boundary complex of the $d$-dimensional cross-polytope (equivalently, the simplicial join of $d$ copies of the 0 -dimensional sphere).

Theorem 1.5. Every flag $(d-1)$-dimensional homology sphere is a vertex-induced (hence quasigeometric and flag) homology subdivision of $\Sigma_{d-1}$.

The proof of Theorem 1.3 relies on the theory of face enumeration for simplicial subdivisions, developed by Stanley [1992]. Given a simplicial complex $\Delta$ and a simplicial subdivision $\Delta^{\prime}$ of $\Delta$, the $h$-vector of $\Delta^{\prime}$ can be expressed in terms of local contributions, one for each face of $\Delta$, and the combinatorics of $\Delta$ [Stanley 1992, Theorem 3.2]. The local contributions are expressed in terms of the key concept of a local $h$-vector, introduced and studied in [Stanley 1992]. When $\Delta$ is Eulerian, this formula transforms into one involving $\gamma$-vectors (Proposition 5.3) and leads to the concept of a local $\gamma$-vector, introduced in Section 5. Using the Davis-Okun theorem [Davis and Okun 2001] mentioned earlier, it is shown that the local $\gamma$-vector has nonnegative coefficients for every flag vertex-induced homology subdivision of the 3-dimensional simplex. Theorem 1.3 is deduced from these results in Section 5.

The proof of Theorem 1.5 is motivated by that of [Athanasiadis 2011, Theorem 1.2], stating that the graph of any flag simplicial pseudomanifold of dimension $d-1$ contains a subdivision of the graph of $\Sigma_{d-1}$.

We now briefly describe the content and structure of this paper. Sections 2 and 3 provide the necessary background on simplicial complexes, subdivisions and their face enumeration. The notion of a homology subdivision, which is convenient for the results of this paper as well as those of a flag subdivision and vertex-induced (a natural strengthening of quasigeometric) subdivision, are introduced in Section 2C. 
Section 3C includes a simple example (see Example 3.4) that shows that there exist quasigeometric subdivisions of the simplex with nonunimodal local $h$-vector.

Section 4 proves Theorem 1.5 and another structural result on flag subdivisions (Proposition 4.6), stating that every flag vertex-induced homology subdivision of the $(d-1)$-dimensional simplex naturally occurs as a restriction of a flag vertex-induced homology subdivision of $\Sigma_{d-1}$. These results are used in Section 5.

Local $\gamma$-vectors are introduced in Section 5, where examples and elementary properties are discussed. It is conjectured there that the local $\gamma$-vector has nonnegative coordinates for every flag vertex-induced homology subdivision of the simplex (Conjecture 5.4). This statement can be considered as a local analogue of Conjecture 1.1. It is shown to imply both Conjectures 1.1 and 1.4 and to hold in dimension 3. Section 5 concludes with the proof of Theorem 1.3.

Section 6 discusses some special cases of Conjecture 5.4. For instance, the conjecture is shown to hold for iterated edge subdivisions (in the sense of [Charney and Davis 1995, Section 5.3]) of the simplex.

\section{Flag complexes, subdivisions and $\gamma$-vectors}

This section reviews background material on simplicial complexes, in particular on their homological properties and subdivisions. For more information on these topics, the reader is referred to [Stanley 1996]. Throughout this paper, $k$ will be a field that we will assume to be fixed. We will denote by $|S|$ the cardinality, and by $2^{S}$ the set of all subsets, of a finite set $S$.

2A. Simplicial complexes. All simplicial complexes we consider will be abstract and finite. Thus, given a finite set $\Omega$, a simplicial complex on the ground set $\Omega$ is a collection $\Delta$ of subsets of $\Omega$ such that $F \subseteq G \in \Delta$ implies $F \in \Delta$. The elements of $\Delta$ are called faces. The dimension of a face $F$ is defined as one less than the cardinality of $F$. The dimension of $\Delta$ is the maximum dimension of a face and is denoted by $\operatorname{dim}(\Delta)$. Faces of $\Delta$ of dimension 0 or 1 are called vertices or edges, respectively. A facet of $\Delta$ is a face that is maximal with respect to inclusion. The complex $\Delta$ is called pure if all its facets have the same dimension. All topological properties of $\Delta$ we mention in the sequel will refer to those of the geometric realization $\|\Delta\|$ of $\Delta$ [Björner 1995, Section 9], uniquely defined up to homeomorphism. For example, we say that $\Delta$ is a simplicial or topological ball or sphere if $\|\Delta\|$ is homeomorphic to a ball or sphere, respectively.

The open star of a face $F \in \Delta$, denoted by st $\Delta(F)$, is the collection of all faces of $\Delta$ that contain $F$. The closed star of $F \in \Delta$, denoted by $\overline{s t}_{\Delta}(F)$, is the subcomplex of $\Delta$ consisting of all subsets of the elements of $\operatorname{st}_{\Delta}(F)$. The link of the face $F \in \Delta$ is the subcomplex of $\Delta$ defined as $\operatorname{link}_{\Delta}(F)=\{G \backslash F: G \in \Delta, F \subseteq G\}$. The simplicial join $\Delta_{1} * \Delta_{2}$ of two collections $\Delta_{1}$ and $\Delta_{2}$ of subsets of disjoint ground 
sets is the collection whose elements are the sets of the form $F_{1} \cup F_{2}$, where $F_{1} \in \Delta_{1}$ and $F_{2} \in \Delta_{2}$. If $\Delta_{1}$ and $\Delta_{2}$ are simplicial complexes, then so is $\Delta_{1} * \Delta_{2}$. The simplicial join of $\Delta$ with the zero-dimensional complex $\{\varnothing,\{v\}\}$ is denoted by $v * \Delta$ and called the cone over $\Delta$ on the (new) vertex $v$.

A simplicial complex $\Delta$ is called flag if every minimal nonface of $\Delta$ has two elements. The closed star, the link of any face of a flag complex and the simplicial join of two (or more) flag complexes are also flag complexes. In particular, the simplicial join of $d$ copies of the zero-dimensional complex with two vertices is a flag complex (in fact, a flag triangulation of the $(d-1)$-dimensional sphere), which will be denoted by $\Sigma_{d-1}$. Explicitly, $\Sigma_{d-1}$ can be described as the simplicial complex on the $2 d$-element ground set $\Omega_{d}=\left\{u_{1}, u_{2}, \ldots, u_{d}\right\} \cup\left\{v_{1}, v_{2}, \ldots, v_{d}\right\}$ whose faces are those subsets of $\Omega_{d}$ that contain at most one element from each of the sets $\left\{u_{i}, v_{i}\right\}$ for $1 \leq i \leq d$.

2B. Homology balls and spheres. Let $\Delta$ be a simplicial complex of dimension $d-1$. We call $\Delta$ a homology sphere (over $\mathbb{k}$ ) if for every $F \in \Delta$ (including $F=\varnothing$ ) we have

$$
\widetilde{H}_{i}\left(\operatorname{link}_{\Delta}(F), \mathbb{k}\right)= \begin{cases}\mathbb{k} & \text { if } i=\operatorname{dim}_{\operatorname{link}}(F), \\ 0 & \text { otherwise }\end{cases}
$$

where $\widetilde{H}_{*}(\Gamma, \mathbb{k})$ denotes reduced simplicial homology of $\Gamma$ with coefficients in $\mathbb{k}$. We call $\Delta$ a homology ball (over $\mathbb{k}$ ) if there exists a subcomplex $\partial \Delta$ of $\Delta$, called the boundary of $\Delta$, so that the following hold:

- $\partial \Delta$ is a $(d-2)$-dimensional homology sphere over $\mathbb{k}$.

- For every $F \in \Delta$ (including $F=\varnothing$ ) we have

$$
\widetilde{H}_{i}\left(\operatorname{link}_{\Delta}(F), \mathbb{k}\right)= \begin{cases}\mathbb{k} & \text { if } F \notin \partial \Delta \text { and } i=\operatorname{dim}_{\operatorname{link}}(F), \\ 0 & \text { otherwise. }\end{cases}
$$

The interior of $\Delta$ is defined as $\operatorname{int}(\Delta)=\Delta$ if $\Delta$ is a homology sphere and as $\operatorname{int}(\Delta)=\Delta \backslash \partial \Delta$ if $\Delta$ is a homology ball. For example, the simplicial complex $\{\varnothing,\{v\}\}$ with a unique vertex $v$ is a 0 -dimensional homology ball (over any field) with boundary $\{\varnothing\}$ and interior $\{\{v\}\}$. If $\Delta$ is a homology ball of dimension $d-1$, then $\partial \Delta$ consists exactly of the faces of those $(d-2)$-dimensional faces of $\Delta$ that are contained in a unique facet of $\Delta$.

Remark 2.1. It follows from standard facts [Björner 1995, (9.12)] on the homology of simplicial joins that the simplicial join of a homology sphere and a homology ball or of two homology balls is a homology ball and that the simplicial join of two homology spheres is again a homology sphere. Moreover, in each case the interior of the simplicial join is equal to the simplicial join of the interiors of the two complexes in question. 
2C. Subdivisions. We will adopt the following notion of homology subdivision of an abstract simplicial complex. This notion generalizes that of topological subdivision of [Stanley 1992, Section 2]. We should point out that the class of homology subdivisions of simplicial complexes is contained in the much broader class of formal subdivisions of Eulerian posets, introduced and studied in [Stanley 1992, Section 7].

Definition 2.2. Let $\Delta$ be a simplicial complex. A (finite, simplicial) homology subdivision of $\Delta$ (over $\mathbb{k}$ ) is a simplicial complex $\Delta^{\prime}$ together with a map $\sigma: \Delta^{\prime} \rightarrow \Delta$ such that the following hold for every $F \in \Delta$ : (a) the set $\Delta_{F}^{\prime}=\sigma^{-1}\left(2^{F}\right)$ is a subcomplex of $\Delta^{\prime}$ that is a homology ball (over $\mathbb{k}$ ) of $\operatorname{dimension} \operatorname{dim}(F)$, and (b) $\sigma^{-1}(F)$ consists of the interior faces of $\Delta_{F}^{\prime}$.

Such a map $\sigma$ is said to be a topological subdivision if the complex $\Delta_{F}^{\prime}$ is homeomorphic to a ball of dimension $\operatorname{dim}(F)$ for every $F \in \Delta$.

Let $\sigma: \Delta^{\prime} \rightarrow \Delta$ be a homology subdivision of $\Delta$. From the defining properties, it follows that the map $\sigma$ is surjective and that $\operatorname{dim}(\sigma(E)) \geq \operatorname{dim}(E)$ for every $E \in \Delta^{\prime}$. Given faces $E \in \Delta^{\prime}$ and $F \in \Delta$, the face $\sigma(E)$ of $\Delta$ is called the carrier of $E$; the subcomplex $\Delta_{F}^{\prime}$ is called the restriction of $\Delta^{\prime}$ to $F$. The subdivision $\sigma$ is called quasigeometric [Stanley 1992, Definition 4.1(a)] if there do not exist $E \in \Delta^{\prime}$ and face $F \in \Delta$ of dimension smaller than $\operatorname{dim}(E)$ such that the carrier of every vertex of $E$ is contained in $F$. Moreover, $\sigma$ is called geometric [Stanley 1992, Definition 4.1(b)] if there exists a geometric realization of $\Delta^{\prime}$ that geometrically subdivides a geometric realization of $\Delta$ in the way prescribed by $\sigma$.

Clearly, if $\sigma: \Delta^{\prime} \rightarrow \Delta$ is a homology or topological subdivision, then the restriction of $\sigma$ to $\Delta_{F}^{\prime}$ is also a homology or topological subdivision of the simplex $2^{F}$ for every $F \in \Delta$, respectively. Moreover, if $\sigma$ is quasigeometric or geometric, respectively, then so are all its restrictions $\Delta_{F}^{\prime}$ for $F \in \Delta$. As part (c) of the following example shows, the restriction of $\sigma$ to a face $F \in \Delta$ need not be a flag complex even when $\Delta^{\prime}$ and $\Delta$ are flag complexes and $\sigma$ is quasigeometric.

Example 2.3. Consider a 3-dimensional simplex $2^{V}$ with $V=\{a, b, c, d\}$ and set $F=\{b, c, d\}$.

(a) Let $\Gamma$ be the simplicial complex consisting of the subsets of $V$ and the subsets of $\{b, c, d, e\}$, and let $\sigma: \Gamma \rightarrow 2^{V}$ be the subdivision (considered in part (h) of [Stanley 1992, Example 2.3]) that pushes $\Gamma$ into the simplex $2^{V}$ so that the face $F$ of $\Gamma$ ends up in the interior of $2^{V}$ and $e$ ends up in the interior of $2^{F}$. Formally, for $E \in \Gamma$ we let $\sigma(E)=E$ if $E \in 2^{V} \backslash\{F\}$, we let $\sigma(E)=V$ if $E$ contains $F$ and otherwise we let $\sigma(E)=F$. Then $\Gamma$ is a flag complex and the restriction $\Gamma_{F}$ of $\sigma$ is the cone over the boundary of $2^{F}$ (with new vertex $e$ ), which is not flag. See left half of Figure 1. 

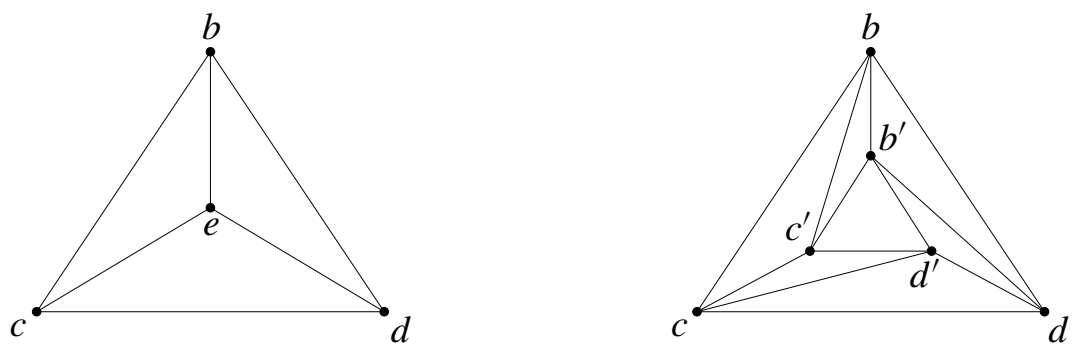

Figure 1. Two nonflag subdivisions of a triangle. Left: see parts (a) and (b) of Example 2.3. Right: see part (c) of Example 2.3.

(b) Let $\Gamma^{\prime}$ be the simplicial complex consisting of the faces of the simplex $2^{V}$ and those of the cone on a vertex $v$ over the boundary of the simplex with vertex set $\{b, c, d, e\}$. (Note that $\Gamma^{\prime}$ is not flag.) Consider the subdivision $\sigma^{\prime}: \Gamma^{\prime} \rightarrow 2^{V}$ that satisfies $\sigma^{\prime}(E)=V$ for every face $E \in \Gamma^{\prime}$ containing $v$ and otherwise agrees with the subdivision $\sigma$ of part (a). Then $\sigma^{\prime}$ is quasigeometric, and its restriction $\Gamma_{F}^{\prime}=\Gamma_{F}$ is again the nonflag complex shown in the left half of Figure 1.

(c) Let $\Gamma_{0}$ be the simplicial complex on the ground set $F \cup\left\{b^{\prime}, c^{\prime}, d^{\prime}\right\}$ whose faces are $F$ and those of the simplicial subdivision of $2^{F}$, shown in Figure 1, right. Let $\Gamma^{\prime \prime}$ consist of the faces of $2^{V}$ and those of the cone over $\Gamma_{0}$ on a new vertex $v$. We leave to the reader to verify that $\Gamma^{\prime \prime}$ is a flag simplicial complex and that it admits a quasigeometric subdivision $\sigma^{\prime \prime}: \Gamma^{\prime \prime} \rightarrow 2^{V}$ (satisfying $\sigma^{\prime \prime}(v)=\sigma^{\prime \prime}(F)=V$ ) for which the restriction $\Gamma_{F}^{\prime \prime}$ is the nonflag simplicial complex shown in Figure 1, right.

The previous examples suggest the following definitions:

Definition 2.4. Let $\Delta^{\prime}$ and $\Delta$ be simplicial complexes, and let $\sigma: \Delta^{\prime} \rightarrow \Delta$ be a homology subdivision.

(i) We say that $\sigma$ is vertex-induced if for all faces $E \in \Delta^{\prime}$ and $F \in \Delta$ the following condition holds: if every vertex of $E$ is a vertex of $\Delta_{F}^{\prime}$, then $E \in \Delta_{F}^{\prime}$.

(ii) We say that $\sigma$ is a flag subdivision if the restriction $\Delta_{F}^{\prime}$ is a flag complex for every face $F \in \Delta$.

For homology or topological subdivisions, we have the hierarchy of properties: geometric $\Rightarrow$ vertex-induced $\Rightarrow$ quasigeometric. The subdivision $\Gamma$ of Example 2.3 is not quasigeometric while $\Gamma^{\prime}$ and $\Gamma^{\prime \prime}$ are quasigeometric but not vertex-induced. (None of the three subdivisions is flag.) Thus, the second implication above is strict. An example discussed in [Chan 1994, p. 468] shows that the first implication is strict as well. We also point out here that if $\sigma: \Delta^{\prime} \rightarrow \Delta$ is a vertex-induced homology subdivision and the simplicial complex $\Delta^{\prime}$ is flag, then $\sigma$ is a flag subdivision. 
Joins and links. The notion of a (vertex-induced or flag) homology subdivision behaves well with respect to simplicial joins and links, as we now explain. Let $\sigma_{1}: \Delta^{\prime}{ }_{1} \rightarrow \Delta_{1}$ and $\sigma_{2}: \Delta^{\prime}{ }_{2} \rightarrow \Delta_{2}$ be homology subdivisions of two simplicial complexes $\Delta_{1}$ and $\Delta_{2}$ on disjoint ground sets. The simplicial join $\Delta^{\prime}{ }_{1} * \Delta^{\prime}{ }_{2}$ is naturally a homology subdivision of $\Delta_{1} * \Delta_{2}$ with subdivision map $\sigma: \Delta^{\prime}{ }_{1} * \Delta^{\prime}{ }_{2} \rightarrow \Delta_{1} * \Delta_{2}$ defined by $\sigma\left(E_{1} \cup E_{2}\right)=\sigma_{1}\left(E_{1}\right) \cup \sigma_{2}\left(E_{2}\right)$ for $E_{1} \in \Delta^{\prime}{ }_{1}$ and $E_{2} \in \Delta^{\prime}{ }_{2}$. Indeed, given faces $F_{1} \in \Delta_{1}$ and $F_{2} \in \Delta_{2}$, the restriction of $\Delta^{\prime}{ }_{1} * \Delta^{\prime}{ }_{2}$ to the face $F=F_{1} \cup F_{2} \in$ $\Delta_{1} * \Delta_{2}$ is equal to $\left(\Delta^{\prime}{ }_{1}\right)_{F_{1}} *\left(\Delta^{\prime}{ }_{2}\right)_{F_{2}}$, which, by Remark 2.1, is a homology ball of dimension equal to that of $F_{1} \cup F_{2}$. Moreover, $\sigma^{-1}(F)=\sigma_{1}^{-1}\left(F_{1}\right) * \sigma_{2}^{-1}\left(F_{2}\right)$, and hence, $\sigma^{-1}(F)$ is the interior of this ball.

Similarly, let $\sigma: \Delta^{\prime} \rightarrow \Delta$ be a homology subdivision, and let $F$ be a common face of $\Delta$ and $\Delta^{\prime}$ (such as a vertex of $\Delta$ ) that satisfies $\sigma(F)=F$. An easy application of part (ii) of Lemma 4.1 shows that $\operatorname{link}_{\Delta^{\prime}}(F)$ is a homology subdivision of $\operatorname{link}_{\Delta}(F)$ with subdivision map $\sigma_{F}$ : $\operatorname{link}_{\Delta^{\prime}}(F) \rightarrow \operatorname{link}_{\Delta}(F)$ defined by $\sigma_{F}(E)=\sigma(E \cup F) \backslash F$. We will refer to this subdivision as the link of $\sigma$ at $F$; its restriction to a face

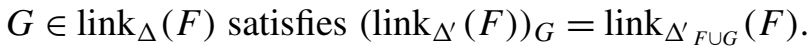

The following statement is an easy consequence of the relevant definitions; the proof is left to the reader:

Lemma 2.5. The simplicial join of two vertex-induced or flag homology subdivisions is also vertex-induced or flag, respectively. The link of a vertex-induced or flag homology subdivision is also vertex-induced or flag, respectively.

Stellar subdivisions. We recall the following standard way to subdivide a simplicial complex. Given a simplicial complex $\Delta$ on the ground set $\Omega$, a face $F \in \Delta$ and an element $v$ not in $\Omega$, the stellar subdivision of $\Delta$ on $F$ (with new vertex $v$ ) is the simplicial complex

$$
\Delta^{\prime}=\left(\Delta \backslash \mathrm{st}_{\Delta}(F)\right) \cup\left(\{v\} * \partial\left(2^{F}\right) * \operatorname{link}_{\Delta}(F)\right)
$$

on the ground set $\Omega \cup\{v\}$, where $\partial\left(2^{F}\right)=2^{F} \backslash\{F\}$. The map $\sigma: \Delta^{\prime} \rightarrow \Delta$, defined by

$$
\sigma(E)= \begin{cases}E & \text { if } E \in \Delta, \\ (E \backslash\{v\}) \cup F & \text { otherwise }\end{cases}
$$

for $E \in \Delta^{\prime}$, is a topological (and thus a homology) subdivision of $\Delta$. We leave to the reader to check that if $\Delta$ is a flag complex and $F \in \Delta$ is an edge, then the stellar subdivision of $\Delta$ on $F$ is again a flag complex.

\section{Face enumeration, $\boldsymbol{\gamma}$-vectors and local $\boldsymbol{h}$-vectors}

This section reviews the definitions and main properties of the enumerative invariants of simplicial complexes and their subdivisions that will appear in the following sections, namely the $h$-vector of a simplicial complex, the $\gamma$-vector of an Eulerian 
simplicial complex and the local $h$-vector of a simplicial subdivision of a simplex. Some new results on local $h$-vectors are included.

3A. $\boldsymbol{h}$-vectors. A fundamental enumerative invariant of a $(d-1)$-dimensional simplicial complex $\Delta$ is the $h$-polynomial, defined by

$$
h(\Delta, x)=\sum_{F \in \Delta} x^{|F|}(1-x)^{d-|F|} .
$$

The $h$-vector of $\Delta$ is the sequence $h(\Delta)=\left(h_{0}(\Delta), h_{1}(\Delta), \ldots, h_{d}(\Delta)\right)$, where $h(\Delta, x)=\sum_{i=0}^{d} h_{i}(\Delta) x^{i}$. The number

$$
(-1)^{d-1} h_{d}(\Delta)=\sum_{F \in \Delta}(-1)^{|F|-1}
$$

is the reduced Euler characteristic of $\Delta$ and is denoted by $\tilde{\chi}(\Delta)$. The polynomial $h(\Delta, x)$ satisfies $h_{i}(\Delta)=h_{d-i}(\Delta)$ [Stanley 1997, Section 3.14] if $\Delta$ is an Eulerian complex, meaning that

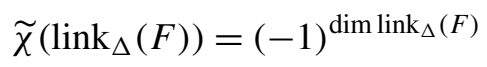

holds for every $F \in \Delta$. For the simplicial join of two simplicial complexes $\Delta_{1}$ and $\Delta_{2}$ we have $h\left(\Delta_{1} * \Delta_{2}, x\right)=h\left(\Delta_{1}, x\right) h\left(\Delta_{2}, x\right)$. For a homology ball or sphere $\Delta$ of dimension $d-1$ we set

$$
h(\operatorname{int}(\Delta), x)=\sum_{F \in \operatorname{int}(\Delta)} x^{|F|}(1-x)^{d-|F|}
$$

and recall the following well known statement (see, for instance, Theorem 7.1 in [Stanley 1996, Chapter II] and [Athanasiadis 2012, Section 2.1] for additional references).

Lemma 3.1. Let $\Delta$ be a $(d-1)$-dimensional simplicial complex. If $\Delta$ is either a homology ball or a homology sphere over $\mathbb{k}$, then $x^{d} h(\Delta, 1 / x)=h(\operatorname{int}(\Delta), x)$.

3B. $\gamma$-vectors. Let $h=\left(h_{0}, h_{1}, \ldots, h_{d}\right)$ be a vector with real coordinates, and let $h(x)=\sum_{i=0}^{d} h_{i} x^{i}$ be the associated real polynomial of degree at most $d$. We say that $h(x)$ has symmetric coefficients and that the vector $h$ is symmetric if $h_{i}=h_{d-i}$ holds for $0 \leq i \leq d$. It is easy to check [Gal 2005, Proposition 2.1.1] that $h(x)$ has symmetric coefficients if and only if there exists a real polynomial $\gamma(x)=\sum_{i=0}^{\lfloor d / 2\rfloor} \gamma_{i} x^{i}$ of degree at most $\lfloor d / 2\rfloor$ satisfying

$$
h(x)=(1+x)^{d} \gamma\left(\frac{x}{(1+x)^{2}}\right)=\sum_{i=0}^{\lfloor d / 2\rfloor} \gamma_{i} x^{i}(1+x)^{d-2 i} .
$$

In that case, $\gamma(x)$ is uniquely determined by $h(x)$ and called the $\gamma$-polynomial 
associated with $h(x)$; the sequence $\left(\gamma_{0}, \gamma_{1}, \ldots, \gamma_{\lfloor d / 2\rfloor}\right)$ is called the $\gamma$-vector associated with $h$. We will refer to the $\gamma$-polynomial associated with the $h$-polynomial of an Eulerian complex $\Delta$ as the $\gamma$-polynomial of $\Delta$ and will denote it by $\gamma(\Delta, x)$. Similarly, we will refer to the $\gamma$-vector associated with the $h$-vector of an Eulerian complex $\Delta$ as the $\gamma$-vector of $\Delta$ and will denote it by $\gamma(\Delta)$.

3C. Local h-vectors. We now recall some of the basics of the theory of face enumeration for subdivisions of simplicial complexes [Stanley 1992; 1996, Section III.10]. The following definition is a restatement of [Stanley 1992, Definition 2.1] for homology (rather than topological) subdivisions of the simplex:

Definition 3.2. Let $V$ be a set with $d$ elements, and let $\Gamma$ be a homology subdivision of the simplex $2^{V}$. The polynomial $\ell_{V}(\Gamma, x)=\ell_{0}+\ell_{1} x+\cdots+\ell_{d} x^{d}$ defined by

$$
\ell_{V}(\Gamma, x)=\sum_{F \subseteq V}(-1)^{d-|F|} h\left(\Gamma_{F}, x\right)
$$

is the local h-polynomial of $\Gamma$ (with respect to $V$ ). The local h-vector of $\Gamma$ (with respect to $V)$ is the sequence $\ell_{V}(\Gamma)=\left(\ell_{0}, \ell_{1}, \ldots, \ell_{d}\right)$.

The following theorem, stated for homology subdivisions, summarizes some of the main properties of local $h$-vectors (see Theorems 3.2 and 3.3 and Corollary 4.7 in [Stanley 1992]):

Theorem 3.3. (i) For every homology subdivision $\Delta^{\prime}$ of a pure simplicial complex $\Delta$ we have

$$
h\left(\Delta^{\prime}, x\right)=\sum_{F \in \Delta} \ell_{F}\left(\Delta_{F}^{\prime}, x\right) h\left(\operatorname{link}_{\Delta}(F), x\right) .
$$

(ii) The local h-vector $\ell_{V}(\Gamma)$ is symmetric for every homology subdivision $\Gamma$ of the simplex $2^{V}$.

(iii) The local h-vector $\ell_{V}(\Gamma)$ has nonnegative coordinates for every quasigeometric homology subdivision $\Gamma$ of the simplex $2^{V}$.

Proof. Parts (i) and (iii) follow from the proofs of Theorems 3.2 and 4.6, respectively, in [Stanley 1992]. Moreover, Lemma 3.1 implies that every homology subdivision of a simplicial complex is a formal subdivision in the sense of [Stanley 1992, Definition 7.4]. Thus, parts (i) and (ii) are special cases of Corollary 7.7 and Theorem 7.8, respectively, in [Stanley 1992].

Example 3.4. The local $h$-polynomial of the subdivision in part (a) of Example 2.3 was computed in [Stanley 1992] as $\ell_{V}(\Gamma, x)=-x^{2}$. This shows that the assumption in Theorem 3.3(iii) that $\Gamma$ is quasigeometric is essential. For part (b) of Example 2.3 we can easily compute that $\ell_{V}\left(\Gamma^{\prime}, x\right)=x+x^{3}$. Since $\Gamma^{\prime}$ is quasigeometric, this disproves [Stanley 1992, Conjecture 5.4] (see also [Chan 1994, Section 6; Stanley 
1996, p. 134]), stating that local $h$-vectors of quasigeometric subdivisions are unimodal.

The previous example suggests the following question:

Question 3.5. Is the local $h$-vector $\ell_{V}(\Gamma)$ unimodal for every vertex-induced homology subdivision $\Gamma$ of the simplex $2^{V}$ ?

We now show that local $h$-vectors also enjoy a locality property. (This will be useful in the proof of Proposition 6.1.)

Proposition 3.6. Let $\sigma: \Gamma \rightarrow 2^{V}$ be a homology subdivision of the simplex $2^{V}$. For every homology subdivision $\Gamma^{\prime}$ of $\Gamma$ we have

$$
\ell_{V}\left(\Gamma^{\prime}, x\right)=\sum_{E \in \Gamma} \ell_{E}\left(\Gamma_{E}^{\prime}, x\right) \ell_{V}(\Gamma, E, x)
$$

where

$$
\ell_{V}(\Gamma, E, x)=\sum_{\sigma(E) \subseteq F \subseteq V}(-1)^{d-|F|} h\left(\operatorname{link}_{\Gamma_{F}}(E), x\right)
$$

for $E \in \Gamma$.

Proof. By assumption, $\Gamma_{F}^{\prime}$ is a homology subdivision of $\Gamma_{F}$ for every $F \subseteq V$. Thus, using the defining Equation (3-2) for $\ell_{V}\left(\Gamma^{\prime}, x\right)$ and (3-3) to expand $h\left(\Gamma_{F}^{\prime}, x\right)$ for $F \subseteq V$, we get

$$
\begin{aligned}
\ell_{V}\left(\Gamma^{\prime}, x\right) & =\sum_{F \subseteq V}(-1)^{d-|F|} h\left(\Gamma_{F}^{\prime}, x\right) \\
& =\sum_{F \subseteq V}(-1)^{d-|F|} \sum_{E \in \Gamma_{F}} \ell_{E}\left(\Gamma_{E}^{\prime}, x\right) h\left(\operatorname{link}_{\Gamma_{F}}(E), x\right) \\
& =\sum_{E \in \Gamma} \ell_{E}\left(\Gamma_{E}^{\prime}, x\right) \sum_{F \subseteq V: \sigma(E) \subseteq F}(-1)^{d-|F|} h\left(\operatorname{link}_{\Gamma_{F}}(E), x\right),
\end{aligned}
$$

and the proof follows.

Remark 3.7. We call the polynomial $\ell_{V}(\Gamma, E, x)$ defined by (3-5) the relative local $h$-polynomial of $\Gamma$ (with respect to $V$ ) at $E$. This polynomial reduces to $\ell_{V}(\Gamma, x)$ for $E=\varnothing$ and shares many of the important properties of $\ell_{V}(\Gamma, x)$, established in [Stanley 1992]. For instance, using ideas of [Stanley 1992] and their refinements in [Athanasiadis 2012], one can show that $\ell_{V}(\Gamma, E, x)$ has symmetric coefficients in the sense that

$$
x^{d-|E|} \ell_{V}(\Gamma, E, 1 / x)=\ell_{V}(\Gamma, E, x)
$$

for every homology subdivision $\Gamma$ of $2^{V}$ and $E \in \Gamma$ and that $\ell_{V}(\Gamma, E, x)$ has nonnegative coefficients for every quasigeometric homology subdivision $\Gamma$ of $2^{V}$ and $E \in \Gamma$. As a consequence of the latter statement and (3-4), we have 
$\ell_{V}\left(\Gamma^{\prime}, x\right) \geq \ell_{V}(\Gamma, x)$ for every quasigeometric homology subdivision $\Gamma$ of $2^{V}$ and every quasigeometric homology subdivision $\Gamma^{\prime}$ of $\Gamma$. Since these results will not be used in this paper, detailed proofs will appear elsewhere.

\section{Flag subdivisions of $\Sigma_{d-1}$}

This section proves Theorem 1.5 as well as a result on flag subdivisions of the simplex (Proposition 4.6), which will be used in Section 5.

The following lemma gives several technical properties of homology balls and spheres (over the field $\mathbb{k}$ ). We will only sketch the proof, which is fairly straightforward and uses standard tools from algebraic topology.

Lemma 4.1. (i) If $\Delta$ is a homology sphere or ball of dimension $d-1$, then $\operatorname{link}_{\Delta}(F)$ is a homology sphere of dimension $d-|F|-1$ for every $F \in \Delta$ or interior face $F \in \Delta$, respectively.

(ii) If $\Delta$ is a homology ball of dimension $d-1$ and $F \in \Delta$ is a boundary face, then $\operatorname{link}_{\Delta}(F)$ is a homology ball of dimension $d-|F|-1$ with interior equal to $\operatorname{link}_{\Delta}(F) \cap \operatorname{int}(\Delta)$.

(iii) If $\Delta$ is a homology sphere or ball, then the cone over $\Delta$ is a homology ball whose boundary is equal to $\Delta$ or the union of $\Delta$ with the cone over the boundary of $\Delta$, respectively.

(iv) Let $\Delta_{1}$ and $\Delta_{2}$ be homology balls of dimension d. If $\Delta_{1} \cap \Delta_{2}$ is a homology ball of dimension $d-1$ that is contained in or equal to the boundary of both $\Delta_{1}$ and $\Delta_{2}$, then $\Delta_{1} \cup \Delta_{2}$ is a homology ball or sphere of dimension d, respectively.

(v) Let $\Delta$ be a homology sphere of dimension $d-1$. If $\Gamma$ is a subcomplex of $\Delta$ that is a homology ball of dimension $d-1$, then the complement of the interior of $\Gamma$ in $\Delta$ is also a homology ball of dimension $d-1$ whose boundary is equal to that of $\Gamma$.

Proof. We first observe that for all faces $F \in \Delta$ and $E \in \operatorname{link}_{\Delta}(F)$, the link of $E$ in $\operatorname{link}_{\Delta}(F)$ is equal to $\operatorname{link}_{\Delta}(E \cup F)$. Moreover, if $\Delta$ is a homology ball and $F$ is an interior face, then so is $E \cup F$. Part (i) follows from these facts and the definition of homology balls and spheres. Part (ii) is an easy consequence of part (i) and the relevant definitions. Part (iii) is an easy consequence of the relevant definitions and the fact that cones have vanishing reduced homology. Part (iv) follows by an easy application of the Mayer-Vietoris long exact sequence [Munkres 1984, §25].

For the last part, we let $\mathrm{K}$ denote the complement of the interior of $\Gamma$ in $\Delta$ and note that the pairs $(\|\Gamma\|,\|\partial \Gamma\|)$ and $(\|\Delta\|,\|\mathrm{K}\|)$ are compact triangulated relative homology manifolds that are orientable over $\mathbb{k}$. Applying the Lefschetz duality theorem [Munkres 1984, §70] and the long exact homology sequence [Munkres 
$1984, \S 23$ ] to these pairs shows that K has trivial reduced homology over $\mathbb{k}$. Similar arguments work for the links of faces of $\mathrm{K}$. The details are omitted.

Remark 4.2. Although not all parts of Lemma 4.1 remain valid if homology balls and spheres are replaced by topological balls and spheres, they do hold for the subclasses of PL balls and PL spheres. (We refer the reader to [Björner et al. 1999, Section 4.7 (d)] for this claim, for a short introduction to PL topology and for additional references.) Thus, the results of this paper remain valid when homology balls and spheres are replaced by PL balls and spheres and the notion of homology subdivision is replaced by its natural PL analogue.

The following lemma will also be essential in the proof of Theorem 1.5. A similar result has appeared in [Barmak 2010, Lemma 3.2].

Lemma 4.3. Let $\Delta$ be a flag $(d-1)$-dimensional homology sphere. For every nonempty face $F$ of $\Delta$, the subcomplex $\bigcup_{v \in F} \overline{s t}_{\Delta}(v)$ is a homology $(d-1)$ dimensional ball whose interior is equal to $\bigcup_{v \in F} \mathrm{st}_{\Delta}(v)$.

Proof. We set $F=\left\{v_{1}, v_{2}, \ldots, v_{k}\right\}, \bigcup_{i=1}^{k} \overline{s t}_{\Delta}\left(v_{i}\right)=\mathrm{K}$ and $\bigcup_{i=1}^{k} \mathrm{st}_{\Delta}\left(v_{i}\right)=\mathrm{L}$ and proceed by induction on the cardinality $k$ of $F$. For $k=1$, the complex $\mathrm{K}$ is the cone over $\operatorname{link}_{\Delta}\left(v_{1}\right)$ on the vertex $v_{1}$. Since $\Delta$ is a homology sphere, the result follows from parts (i) and (iii) of Lemma 4.1. Suppose that $k \geq 2$. We will also assume that $d \geq 3$ since the result is trivial otherwise. (We note that the assumption that $\Delta$ is flag is essential in the case $d=2$.) Since by Lemma 4.1(i) links of flag homology spheres are also flag homology spheres, the complex $\Gamma=\operatorname{link}_{\Delta}\left(v_{k}\right)$ is a flag homology sphere of dimension $d-2$ and $\left\{v_{1}, \ldots, v_{k-1}\right\}$ is a nonempty face of $\Gamma$. Thus, by the induction hypothesis, the union $\Gamma_{1}=\bigcup_{i=1}^{k-1} \overline{s t}_{\Gamma}\left(v_{i}\right)$ is a homology ball of dimension $d-2$. Let $\Gamma_{0}$ denote the boundary of $\Gamma_{1}$, and let $\Gamma_{2}$ denote the complement of the interior of $\Gamma_{1}$ in $\Gamma$. Thus, $\Gamma_{0}$ is a homology sphere of dimension $d-3$, and, by part (v) of Lemma 4.1, $\Gamma_{2}$ is a homology ball of dimension $d-2$ whose boundary is equal to $\Gamma_{0}$.

Consider the union $\mathrm{K}_{1}=\bigcup_{i=1}^{k-1} \overline{s t}_{\Delta}\left(v_{i}\right)$ and the cones $\mathrm{K}_{2}=v_{k} * \Gamma_{2}$ and $\mathrm{K}_{0}=v_{k} * \Gamma_{0}$. It is straightforward to verify that $K=K_{1} \cup K_{2}$ and that $K_{1} \cap K_{2}=K_{0}$. We note that $\mathrm{K}_{1}$ is a homology ball of dimension $d-1$ by the induction hypothesis and that $\mathrm{K}_{2}$ and $\mathrm{K}_{0}$ are homology balls of dimension $d-1$ and $d-2$, respectively, by part (iii) of Lemma 4.1. By the induction hypothesis, the interior of $\Gamma_{1}$ is equal to $\bigcup_{i=1}^{k-1} \operatorname{st}_{\Gamma}\left(v_{i}\right)$. Therefore, none of the faces of $\Gamma_{0}$ contains any of $v_{1}, \ldots, v_{k-1}$, and hence, the same holds for $\mathrm{K}_{0}$. Since by the induction hypothesis the interior of $\mathrm{K}_{1}$ is equal to $\bigcup_{i=1}^{k-1} \operatorname{st}_{\Delta}\left(v_{i}\right)$, we conclude that $\mathrm{K}_{0}$ is contained in the boundary of $\mathrm{K}_{1}$. Moreover, $\mathrm{K}_{0}$ is also contained in the boundary of $\mathrm{K}_{2}$ since $\Gamma_{0}$ is contained in the boundary of $\Gamma_{2}$. It follows from the previous discussion and Lemma 4.1(iv) that $\mathrm{K}$ is a homology $(d-1)$-dimensional ball. 
We now verify that the interior of $\mathrm{K}$ is equal to $\mathrm{L}$. This statement may be derived from the previous inductive argument since the interior of $\mathrm{K}$ is equal to the union of the interiors of $\mathrm{K}_{1}, \mathrm{~K}_{2}$ and $\mathrm{K}_{0}$. We give the following alternative argument: Since $\mathrm{K}$ is a homology ball, its boundary consists of all faces of the $(d-2)$-dimensional faces of $\mathrm{K}$ that are contained in exactly one facet of $\mathrm{K}$. The validity of the statement for $k=1$ implies that these $(d-2)$-dimensional faces of $\mathrm{K}$ are precisely those that do not contain any of the $v_{i}$ and that are not contained in more than one of the subcomplexes $\operatorname{link}_{\Delta}\left(v_{i}\right)$. However, since $\Delta$ is $(d-1)$-dimensional and flag, no $(d-2)$-dimensional face of $\Delta$ may be contained in more than one of the link ${ }_{\Delta}\left(v_{i}\right)$. Thus, the boundary of $\mathrm{K}$ consists precisely of its faces that do not contain any of the $v_{i}$, and the proof follows.

Proof of Theorem 1.5. Let $\Delta$ be a flag simplicial complex of dimension $d-1$ and $\Sigma_{d-1}$ be the simplicial join of the zero-dimensional spheres $\left\{\varnothing,\left\{u_{i}\right\},\left\{v_{i}\right\}\right\}$ for $1 \leq i \leq d$. We fix a facet $\left\{x_{1}, x_{2}, \ldots, x_{d}\right\}$ of $\Delta$, and for $E \in \Delta$ we define

$$
\sigma(E)=\left\{u_{i}: x_{i} \in E\right\} \cup\left\{v_{i}: E \notin \overline{\mathrm{st}}_{\Delta}\left(x_{i}\right)\right\} .
$$

Clearly, $\sigma(E)$ cannot contain any of the sets $\left\{u_{i}, v_{i}\right\}$. Thus, we have $\sigma(E) \in \Sigma_{d-1}$ for every $E \in \Delta$, and hence, we get a map $\sigma: \Delta \rightarrow \Sigma_{d-1}$. We will prove that this map is a homology subdivision of $\Sigma_{d-1}$ if $\Delta$ is a homology sphere. Given a face $F \in \Sigma_{d-1}$, we need to show that $\sigma^{-1}\left(2^{F}\right)$ is a subcomplex of $\Delta$ of $\operatorname{dimension} \operatorname{dim}(F)$ that is a homology ball with interior $\sigma^{-1}(F)$. We denote by $S$ the subset of $\left\{x_{1}, x_{2}, \ldots, x_{d}\right\}$ consisting of all vertices $x_{i}$ for which $F \cap\left\{u_{i}, v_{i}\right\}=\varnothing$ and distinguish two cases:

Case 1: $S=\varnothing$. We may assume that $F=\left\{u_{1}, \ldots, u_{k}\right\} \cup\left\{v_{k+1}, \ldots, v_{d}\right\}$ for some $k \leq d$. Setting $E_{0}=\left\{x_{1}, \ldots, x_{k}\right\}$, the defining Equation (4-1) shows that $\sigma^{-1}\left(2^{F}\right)$ is equal to the intersection of $\bigcap_{i=1}^{k} \overline{\mathrm{st}}_{\Delta}\left(x_{i}\right)$ with the complement of $\bigcup_{i=k+1}^{d} \mathrm{st}_{\Delta}\left(x_{i}\right)$ in $\Delta$ and that $\sigma^{-1}(F)$ consists of those faces of $\sigma^{-1}\left(2^{F}\right)$ that contain $E_{0}$ and do not belong to any of the $\operatorname{link}_{\Delta}\left(x_{i}\right)$ for $k+1 \leq i \leq d$. Consider the complex $\Gamma=\operatorname{link}_{\Delta}\left(E_{0}\right)$, and let $\mathrm{K}$ denote the complement of the union $\bigcup_{i=k+1}^{d} \mathrm{st}_{\Gamma}\left(x_{i}\right)$ in $\Gamma$. Since links of homology spheres are also homology spheres (see part (i) of Lemma 4.1), the complex $\Gamma$ is a homology sphere of dimension $d-|F|-1$. By Lemma 4.3 and part (v) of Lemma 4.1, $\mathrm{K}$ is a homology ball of dimension $d-|F|-1$ whose interior is equal to the set of those faces of $\mathrm{K}$ that do not belong to any of the $\operatorname{link}_{\Gamma}\left(x_{i}\right)$ for $k+1 \leq i \leq d$. From the above we conclude that $\sigma^{-1}\left(2^{F}\right)$ is equal to the simplicial join of the simplex $2^{E_{0}}$ and $\mathrm{K}$ and that $\sigma^{-1}(F)$ is equal to the simplicial join of $\left\{E_{0}\right\}$ and the interior of $\mathrm{K}$. The result now follows from part (iii) of Lemma 4.1 and the previous discussion.

Case 2: $S \neq \varnothing$. Then $\sigma^{-1}\left(2^{F}\right)$ is contained in $\operatorname{link}_{\Delta}(S)$. As a result, replacing $\Delta$ by $\operatorname{link}_{\Delta}(S)$ reduces this case to the previous one. 
Finally, we note that (4-1) may be rewritten as $\sigma(E)=\bigcup_{x \in E} f(x)$, where

$$
f(x)= \begin{cases}\left\{u_{i}\right\} & \text { if } x=x_{i} \text { for some } 1 \leq i \leq d, \\ \left\{v_{i}: x \notin \operatorname{link}_{\Delta}\left(x_{i}\right)\right\} & \text { otherwise }\end{cases}
$$

for every vertex $x$ of $\Delta$. This implies that for every $E \in \Delta$, the carrier of $E$ is equal to the union of the carriers of the vertices of $E$. As a result, $\sigma$ is vertex-induced and the proof follows.

Corollary 4.4. Given any flag homology sphere $\Delta$ of dimension $d-1$, there exist simplicial complexes $\Gamma_{F}$, one for each face $F \in \Sigma_{d-1}$, with the following properties: (a) $\Gamma_{F}$ is a flag vertex-induced homology subdivision of the simplex $2^{F}$ for every $F \in \Sigma_{d-1}$, and (b) we have

$$
h(\Delta, x)=\sum_{F \in \Sigma_{d-1}} \ell_{F}\left(\Gamma_{F}, x\right)(1+x)^{d-|F|} .
$$

Proof. We apply (3-3) to the subdivision of $\Delta$ guaranteed by Theorem 1.5 and note that for every $F \in \Sigma_{d-1}$, the restriction $\Gamma_{F}$ of this subdivision to $F$ has the required properties and that $h\left(\operatorname{link}_{\Sigma_{d-1}}(F), x\right)=(1+x)^{d-|F|}$.

Remark 4.5. Due to (4-2) and Theorem 3.3(iii), $h(\Delta, x) \geq(1+x)^{d}$ for every flag $(d-1)$-dimensional homology sphere $\Delta$. This inequality was proved, more generally, for every flag $(d-1)$-dimensional doubly Cohen-Macaulay simplicial complex $\Delta$ in [Athanasiadis 2011, Theorem 1.3].

We now fix a $d$-element set $V=\left\{v_{1}, v_{2}, \ldots, v_{d}\right\}$ and a homology subdivision $\Gamma$ of $2^{V}$ with subdivision map $\sigma: \Gamma \rightarrow 2^{V}$. We let $U=\left\{u_{1}, u_{2}, \ldots, u_{d}\right\}$ be a $d$-element set that is disjoint from $V$ and consider the union $\Delta$ of all collections of the form $2^{E} * \Gamma_{G}$, where $E=\left\{u_{i}: i \in I\right\}$ and $G=\left\{v_{j}: j \in J\right\}$ are subsets of $U$ and $V$, respectively, and $(I, J)$ ranges over all ordered pairs of disjoint subsets of $\{1,2, \ldots, d\}$. Clearly, $\Delta$ is a simplicial complex that contains as a subcomplex $\Gamma$ (set $I=\varnothing$ ) and the simplex $2^{U}$ (set $J=\varnothing$ ).

We let $\Sigma_{d-1}$ be as in the proof of Theorem 1.5 and define the map $\sigma_{0}: \Delta \rightarrow \Sigma_{d-1}$ by $\sigma_{0}(E \cup F)=E \cup \sigma(F)$ for all $E \subseteq U$ and $F \in \Gamma$ such that $E \cup F \in \Delta$. The second result of this section is as follows:

Proposition 4.6. Under the established assumptions and notation, we have:

(i) The complex $\Delta$ is a $(d-1)$-dimensional homology sphere.

(ii) Endowed with the map $\sigma_{0}$, the complex $\Delta$ is a homology subdivision of $\Sigma_{d-1}$.

(iii) If $\Gamma$ is flag and vertex-induced, then $\Delta$ is a flag simplicial complex and a flag, vertex-induced homology subdivision of $\Sigma_{d-1}$.

Proof. We first verify (ii). We consider any face $W \in \Sigma_{d-1}$ so that $W=E \cup G$ for some $E \subseteq U$ and $G \subseteq V$ and recall that $\Gamma_{G}$ is a homology ball of dimension $\operatorname{dim}(G)$. 
We have $\sigma_{0}^{-1}\left(2^{W}\right)=2^{E} * \Gamma_{G}$ and $\sigma_{0}^{-1}(W)=\{E\} * \sigma^{-1}(G)=\{E\} * \operatorname{int}\left(\Gamma_{G}\right)$ by the definition of $\sigma_{0}$. Thus, it follows from part (iii) of Lemma 4.1 that $\sigma_{0}^{-1}\left(2^{W}\right)$ is a homology ball of dimension $\operatorname{dim}(W)$ and that its interior is equal to $\sigma_{0}^{-1}(W)$.

Part (i) may be deduced from part (ii) as follows. Let $F_{0}, F_{1}, \ldots, F_{m}$ be a linear ordering of the facets of $\Sigma_{d-1}$ such that $F_{i} \cap U \subset F_{j} \cap U$ implies $i<j$. Thus, we have $m=2^{d}, F_{0}=V$ and $F_{m}=U$. By assumption, $\Delta_{F_{0}}=\Gamma_{V}$ is a $(d-1)$ dimensional homology ball. Moreover, $\Delta_{F_{j}}$ is equal to the simplicial join of a face of $2^{U}$ with the restriction of $\Gamma$ to a face of $2^{V}$ for $1 \leq j \leq m$ and hence a $(d-1)$-dimensional homology ball by part (iii) of Lemma 4.1, and $\Delta_{F_{j}} \cap \bigcup_{i=0}^{j-1} \Delta_{F_{i}}$ is equal to the simplicial join of the boundary of this face with the same restriction of $\Gamma$. It follows from part (iv) of Lemma 4.1 by induction on $j$ that $\bigcup_{i=0}^{j} \Delta_{F_{i}}$ is a $(d-1)$-dimensional homology ball for $0 \leq j \leq m-1$ and a $(d-1)$-dimensional homology sphere for $j=m$. This proves (i) since $\Delta=\bigcup_{i=0}^{m} \Delta_{F_{i}}$.

To verify (iii), assume that $\Gamma$ is flag and vertex-induced. It is clear from the definition of $\sigma_{0}$ that the subdivision $\Delta$ is also vertex-induced. Since the restriction of $\Delta$ to any face of $\Sigma_{d-1}$ is the join of a simplex with the restriction of $\Gamma$ to a face of $2^{V}$, the subdivision $\Delta$ is flag as well. To verify that $\Delta$ is a flag complex, let $E \cup F$ be a set of vertices of $\Delta$ that are pairwise joined by edges, where $E=\left\{u_{i}: i \in I\right\}$ for some $I \subseteq\{1,2, \ldots, d\}$ and $F$ consists of vertices of $\Gamma$. We need to show that $E \cup F \in \Delta$. We set $J=\{1,2, \ldots, d\} \backslash I$ and $G=\left\{v_{j}: j \in J\right\}$ and note that the elements of $F$ are vertices of $\Gamma_{G}$ by definition of $\Delta$. Since the elements of $F$ are pairwise joined by edges in $\Gamma$, our assumptions that $\Gamma$ is vertex-induced and flag imply that $F \in \Gamma_{G}$. Therefore, $E \cup F$ belongs to $2^{E} * \Gamma_{G}$, which is contained in $\Delta$, and the result follows.

Remark 4.7. The conclusion in Proposition 4.6 that $\Delta$ is a flag complex does not hold under the weaker hypothesis that $\Gamma$ is quasigeometric rather than vertexinduced. For instance, let $\Gamma$ be the simplicial complex consisting of the subsets of $V=\left\{v_{1}, v_{2}, v_{3}\right\}$ and $\left\{v_{2}, v_{3}, v_{4}\right\}$, and let $\sigma: \Gamma \rightarrow 2^{V}$ be the subdivision that pushes $\Gamma$ into $2^{V}$ so that the face $F=\left\{v_{2}, v_{3}\right\}$ of $\Gamma$ ends up in the interior of $2^{V}$ and $v_{4}$ ends up in the interior of $2^{F}$. Then $\Gamma$ is quasigeometric and flag, but the simplicial complex $\Delta$ is not flag since it has $\left\{u_{1}, v_{2}, v_{3}\right\}$ as a minimal nonface.

\section{Local $\gamma$-vectors}

This section defines the local $\gamma$-vector of a homology subdivision of the simplex, lists examples and elementary properties, discusses its nonnegativity in the special case of flag subdivisions and concludes with the proof of Theorem 1.3. This proof comes as an application of the considerations and results of the present and the previous sections. 
Definition 5.1. Let $V$ be a set with $d$ elements, and let $\Gamma$ be a homology subdivision of the simplex $2^{V}$. The polynomial $\xi_{V}(\Gamma, x)=\xi_{0}+\xi_{1} x+\cdots+\xi_{\lfloor d / 2\rfloor} x^{\lfloor d / 2\rfloor}$ defined by

$$
\ell_{V}(\Gamma, x)=(1+x)^{d} \xi_{V}\left(\Gamma, \frac{x}{(1+x)^{2}}\right)=\sum_{i=0}^{\lfloor d / 2\rfloor} \xi_{i} x^{i}(1+x)^{d-2 i}
$$

is the local $\gamma$-polynomial of $\Gamma$ (with respect to $V$ ). The local $\gamma$-vector of $\Gamma$ (with respect to $V)$ is the sequence $\xi_{V}(\Gamma)=\left(\xi_{0}, \xi_{1}, \ldots, \xi_{\lfloor d / 2\rfloor}\right)$.

Thus, $\xi_{V}(\Gamma, x)$ is the $\gamma$-polynomial associated with $\ell_{V}(\Gamma, x)$, and $\xi_{V}(\Gamma)$ is the $\gamma$-vector associated with $\ell_{V}(\Gamma)$ in the sense of Section 3B. All formulas in the next example follow from corresponding formulas in [Stanley 1992, Example 2.3] or directly from the relevant definitions.

Example 5.2. (a) For the trivial subdivision $\Gamma=2^{V}$ of the $(d-1)$-dimensional simplex $2^{V}$ we have

$$
\xi_{V}(\Gamma, x)= \begin{cases}1 & \text { if } d=0 \\ 0 & \text { if } d \geq 1\end{cases}
$$

(b) Let $\xi_{V}(\Gamma)=\left(\xi_{0}, \xi_{1}, \ldots, \xi_{\lfloor d / 2\rfloor}\right)$, where $\Gamma$ and $V$ are as in Definition 5.1. Assuming that $d \geq 1$, we have $\xi_{0}=0$ and $\xi_{1}=f_{0}^{\circ}$, where $f_{0}^{\circ}$ is the number of interior vertices of $\Gamma$. Assuming that $d \geq 4$, we also have $\xi_{2}=-(2 d-3) f_{0}^{\circ}+f_{1}^{\circ}-\tilde{f}_{0}$, where $f_{1}^{\circ}$ is the number of interior edges of $\Gamma$ and $\tilde{f}_{0}$ is the number of vertices of $\Gamma$ that lie in the relative interior of a $(d-2)$-dimensional face of $2^{V}$.

(c) Suppose that $d \in\{2,3\}$. As a consequence of (b) we have $\xi_{V}(\Gamma, x)=t x$ for every homology subdivision $\Gamma$ of $2^{V}$, where $t$ is the number of interior vertices of $\Gamma$.

(d) Let $\Gamma$ be the cone over the boundary $2^{V} \backslash\{V\}$ of the simplex $2^{V}$ (so $\Gamma$ is the stellar subdivision of $2^{V}$ on the face $\left.V\right)$. Then $\ell_{V}(\Gamma, x)=x+x^{2}+\cdots+x^{d-1}$, and hence, $\xi_{2}$ is negative for $d \geq 4$. For instance, we have $\xi_{V}(\Gamma, x)=x-x^{2}$ for $d=4$.

(e) For the subdivisions of parts (b) and (c) of Example 2.3 we can compute that $\ell_{V}\left(\Gamma^{\prime}, x\right)=\ell_{V}\left(\Gamma^{\prime \prime}, x\right)=x+x^{3}$ and hence that $\xi_{V}\left(\Gamma^{\prime}, x\right)=\xi_{V}\left(\Gamma^{\prime \prime}, x\right)=x-2 x^{2}$.

The following proposition shows the relevance of local $\gamma$-vectors in the study of $\gamma$-vectors of subdivisions of Eulerian complexes:

Proposition 5.3. Let $\Delta$ be a pure Eulerian simplicial complex. For every homology subdivision $\Delta^{\prime}$ of $\Delta$ we have

$$
\gamma\left(\Delta^{\prime}, x\right)=\sum_{F \in \Delta} \xi_{F}\left(\Delta_{F}^{\prime}, x\right) \gamma\left(\operatorname{link}_{\Delta}(F), x\right) .
$$

Proof. Since $\Delta$ is Eulerian, so is $\operatorname{link}_{\Delta}(F)$ for every $F \in \Delta$. Thus, applying (3-1) 
to the $h$-polynomial of $\operatorname{link}_{\Delta}(F)$ we get

$$
h\left(\operatorname{link}_{\Delta}(F), x\right)=(1+x)^{d-|F|} \gamma\left(\operatorname{link}_{\Delta}(F), \frac{x}{(1+x)^{2}}\right),
$$

where $d-1=\operatorname{dim}(\Delta)$. Using this and (5-1), Equation (3-3) may be rewritten as

$$
h\left(\Delta^{\prime}, x\right)=(1+x)^{d} \sum_{F \in \Delta} \xi_{F}\left(\Delta_{F}^{\prime}, \frac{x}{(1+x)^{2}}\right) \gamma\left(\operatorname{link}_{\Delta}(F), \frac{x}{(1+x)^{2}}\right) .
$$

The proposed equality now follows from the uniqueness of the $\gamma$-polynomial associated with $h\left(\Delta^{\prime}, x\right)$.

The following statement is the main conjecture of this paper:

Conjecture 5.4. For every flag vertex-induced homology subdivision $\Gamma$ of the simplex $2^{V}$ we have $\xi_{V}(\Gamma) \geq 0$.

Parts (d) and (e) of Example 5.2 show that the conclusion of Conjecture 5.4 fails under various weakenings of the hypotheses. We do not know of an example of a flag quasigeometric homology subdivision of the simplex for which the local $\gamma$-vector fails to be nonnegative.

We now discuss some consequences of Theorem 1.5 and Proposition 5.3 related to Conjecture 5.4.

Corollary 5.5. For every flag homology sphere $\Delta$ of dimension $d-1$ we have

$$
\gamma(\Delta, x)=\sum_{F \in \Sigma_{d-1}} \xi_{F}\left(\Gamma_{F}, x\right),
$$

where $\Gamma_{F}$ is as in Corollary 4.4 for each $F \in \Sigma_{d-1}$. In particular, the validity of Conjecture 5.4 for homology subdivisions $\Gamma$ of dimension at most $d-1$ implies the validity of Conjecture 1.1 for homology spheres $\Delta$ of dimension at most $d-1$.

Proof. Setting $\ell_{F}\left(\Gamma_{F}, x\right)=\sum_{i} \xi_{F, i} x^{i}(1+x)^{|F|-2 i}$ in (4-2) and changing the order of summation results in (5-4). Alternatively, one can apply (5-3) to the subdivision guaranteed by Theorem 1.5 and note that $\gamma\left(\operatorname{link}_{\Sigma_{d-1}}(F), x\right)=1$ for every $F \in \Sigma_{d-1}$. The last sentence in the statement of the corollary follows from (5-4).

Corollary 5.6. The validity of Conjecture 5.4 for homology subdivisions $\Gamma$ of dimension at most $d-1$ implies the validity of Conjecture 1.4 for homology spheres $\Delta$ and subdivisions $\Delta^{\prime}$ of dimension at most $d-1$.

Proof. We observe that the term corresponding to $F=\varnothing$ in the sum of the right-hand side of (5-3) is equal to $\gamma(\Delta, x)$. Thus, the result follows from (5-3), Corollary 5.5 and the fact that the link of every nonempty face of a flag homology sphere is also a flag homology sphere of smaller dimension. 
Proposition 5.7. Conjecture 5.4 holds for subdivisions of the 3-dimensional simplex.

Proof. Let $\Gamma$ be a flag vertex-induced homology subdivision of the $(d-1)$ dimensional simplex $2^{V}$, and let $\Delta$ be the homology subdivision of $\Sigma_{d-1}$ considered in Proposition 4.6. Applying (5-3) to this subdivision and noting that $\gamma\left(\operatorname{link}_{\Sigma_{d-1}}(F), x\right)=1$ for every $F \in \Sigma_{d-1}$, we get

$$
\gamma(\Delta, x)=\sum_{F \in \Sigma_{d-1}} \xi_{F}\left(\Delta_{F}, x\right)
$$

By definition of $\Delta$, the restriction $\Delta_{F}$ is a cone over the restriction of $\Delta$ to a proper face of $F$ for every $F \in \Sigma_{d-1}$ that is not contained in $V$. Since every such subdivision has a zero local $h$-vector [Stanley 1992, p. 821], the previous formula can be rewritten as

$$
\gamma(\Delta, x)=\sum_{F \subseteq V} \xi_{F}\left(\Gamma_{F}, x\right)
$$

Assume now that $d=4$ so that $\xi(\Gamma, x)=\xi_{0}+\xi_{1} x+\xi_{2} x^{2}$ for some integers $\xi_{0}, \xi_{1}$ and $\xi_{2}$. Since $\xi_{0}=0$ and $\xi_{1} \geq 0$ by part (b) of Example 5.2, it suffices to show that $\xi_{2} \geq 0$. For that, we observe that the only contribution to the coefficient of $x^{2}$ in the right-hand side of (5-5) comes from the term with $F=V$. As a result, $\xi_{2}$ is equal to the coefficient of $x^{2}$ in $\gamma(\Delta, x)$. Since $\Delta$ is a 3-dimensional flag homology sphere (by Proposition 4.6), this coefficient is nonnegative by the Davis-Okun theorem [Davis and Okun 2001, Theorem 11.2.1], and the result follows.

Proof of Theorem 1.3. For 3-dimensional spheres the result is due to Proposition 5.7 and Corollary 5.6. Assume now that $\Delta$ and $\Delta^{\prime}$ have dimension 4. Then we can write $\gamma(\Delta, x)=1+\gamma_{1}(\Delta) x+\gamma_{2}(\Delta) x^{2}$ and $\gamma\left(\Delta^{\prime}, x\right)=1+\gamma_{1}\left(\Delta^{\prime}\right) x+\gamma_{2}\left(\Delta^{\prime}\right) x^{2}$. Since $\gamma_{1}(\Delta)=f_{0}(\Delta)-8$ and $\gamma_{1}\left(\Delta^{\prime}\right)=f_{0}\left(\Delta^{\prime}\right)-8$, where $f_{0}(\Delta)$ and $f_{0}\left(\Delta^{\prime}\right)$ are the number of vertices of $\Delta$ and $\Delta^{\prime}$, respectively, it is clear that $\gamma_{1}\left(\Delta^{\prime}\right) \geq \gamma_{1}(\Delta)$. As the computation in the proof of [Gal 2005, Corollary 2.2.2] shows, we also have

$$
2 \gamma_{2}(\Delta)=\sum_{v \in \operatorname{vert}(\Delta)} \gamma_{2}\left(\operatorname{link}_{\Delta}(v)\right)
$$

where $\operatorname{vert}(\Delta)$ is the set of vertices of $\Delta$. Similarly, we have

$$
2 \gamma_{2}\left(\Delta^{\prime}\right)=\sum_{v^{\prime} \in \operatorname{vert}\left(\Delta^{\prime}\right)} \gamma_{2}\left(\operatorname{link}_{\Delta^{\prime}}\left(v^{\prime}\right)\right)
$$

where we may assume that $\operatorname{vert}(\Delta) \subseteq \operatorname{vert}\left(\Delta^{\prime}\right)$. Since $\operatorname{link}_{\Delta^{\prime}}(v)$ is a flag vertexinduced homology subdivision of $\operatorname{link}_{\Delta}(v)$ for every $v \in \operatorname{vert}(\Delta)$, by Lemma 2.5, we have $\gamma_{2}\left(\operatorname{link}_{\Delta^{\prime}}(v)\right) \geq \gamma_{2}\left(\operatorname{link}_{\Delta}(v)\right)$ by the 3 -dimensional case, treated earlier, for every such vertex $v$. Since $\operatorname{link}_{\Delta^{\prime}}\left(v^{\prime}\right)$ is a 3-dimensional flag homology sphere, we 
also have $\gamma_{2}\left(\operatorname{link}_{\Delta^{\prime}}\left(v^{\prime}\right)\right) \geq 0$ by the Davis-Okun theorem for every $v^{\prime} \in \operatorname{vert}\left(\Delta^{\prime}\right) \backslash$ $\operatorname{vert}(\Delta)$. Hence, $\gamma_{2}\left(\Delta^{\prime}\right) \geq \gamma_{2}(\Delta)$, and the result follows.

Question 5.8. Does $\gamma\left(\Delta^{\prime}\right) \geq \gamma(\Delta)$ hold for every flag homology sphere $\Delta$ and every flag homology subdivision $\Delta^{\prime}$ of $\Delta$ ?

\section{Special cases}

This section provides some evidence in favor of the validity of Conjecture 5.4 other than that provided by Proposition 5.7.

Simplicial joins. Let $\Gamma$ be a homology subdivision of the simplex $2^{V}$ and $\Gamma^{\prime}$ be a homology subdivision of the simplex $2^{V^{\prime}}$, where $V$ and $V^{\prime}$ are disjoint finite sets. Then $\Gamma * \Gamma^{\prime}$ is a homology subdivision of the simplex $2^{V} * 2^{V^{\prime}}=2^{V \cup V^{\prime}}$, and given subsets $F \subseteq V$ and $F^{\prime} \subseteq V^{\prime}$, the restriction of $\Gamma * \Gamma^{\prime}$ to the face $F \cup F^{\prime}$ of this simplex satisfies $\left(\Gamma * \Gamma^{\prime}\right)_{F \cup F^{\prime}}=\Gamma_{F} * \Gamma_{F^{\prime}}^{\prime}$. Since $h\left(\Gamma_{F} * \Gamma_{F^{\prime}}^{\prime}, x\right)=h\left(\Gamma_{F}, x\right) h\left(\Gamma_{F^{\prime}}^{\prime}, x\right)$, the defining Equation (3-2) and a straightforward computation show that

$$
\ell_{V \cup V^{\prime}}\left(\Gamma * \Gamma^{\prime}, x\right)=\ell_{V}(\Gamma, x) \ell_{V^{\prime}}\left(\Gamma^{\prime}, x\right) .
$$

This equation and (5-1) imply that

$$
\xi_{V \cup V^{\prime}}\left(\Gamma * \Gamma^{\prime}, x\right)=\xi_{V}(\Gamma, x) \xi_{V^{\prime}}\left(\Gamma^{\prime}, x\right) .
$$

From the previous formula and Lemma 2.5 we conclude that if $\Gamma$ and $\Gamma^{\prime}$ satisfy the assumptions and the conclusion of Conjecture 5.4, then so does $\Gamma * \Gamma^{\prime}$.

Edge subdivisions. Following [Charney and Davis 1995, Section 5.3], we refer to the stellar subdivision on an edge of a simplicial complex $\Gamma$ as an edge subdivision. As mentioned in Section 3B, flagness of a simplicial complex is preserved by edge subdivisions. The following statement describes a class of flag (geometric) subdivisions of the simplex with nonnegative local $\gamma$-vectors:

Proposition 6.1. For every subdivision $\Gamma$ of the simplex $2^{V}$ that can be obtained from the trivial subdivision by successive edge subdivisions, we have $\xi_{V}(\Gamma) \geq 0$.

Proof. Let $\Gamma$ be a subdivision of $2^{V}$ and $\Gamma^{\prime}$ be the edge subdivision of $\Gamma$ on $e=\{a, b\} \in \Gamma$. Thus, we have $\Gamma^{\prime}=\left(\Gamma \backslash \operatorname{st}_{\Gamma}(e)\right) \cup\left(\{v\} * \partial(e) * \operatorname{link}_{\Gamma}(e)\right)$, where $v$ is the new vertex added and $\partial(e)=\{\varnothing,\{a\},\{b\}\}$.

By appealing to (3-4) and noticing that the right-hand side of this formula vanishes except when $E \in\{\varnothing, e\}$ (or by direct computation), we find that

$$
\ell_{V}\left(\Gamma^{\prime}, x\right)=\ell_{V}(\Gamma, x)+x \ell_{V}(\Gamma, e, x) .
$$

Thus, it suffices to prove the following claim: if the $\gamma$-polynomial corresponding to $\ell_{V}(\Gamma, E, x)$ has nonnegative coefficients for every face $E \in \Gamma$ of positive dimension 
(meaning that $\ell_{V}(\Gamma, E, x)$ can be written as a linear combination of the polynomials $x^{i}(1+x)^{d-|E|-2 i}$ with nonnegative coefficients for every $\left.|E| \geq 2\right)$, then the same holds for $\Gamma^{\prime}$. We consider a face $E \in \Gamma^{\prime}$ of positive dimension and distinguish the following cases. (We note that $E$ cannot contain $e$ and that if $E \in \Gamma$, then the carrier $\sigma(E) \subseteq V$ of $E$ is the same, whether considered with respect to $\Gamma$ or $\Gamma^{\prime}$.)

Case 1: $E \in \Gamma \backslash \operatorname{link}_{\Gamma}(e)$. The links $\operatorname{link}_{\Gamma_{F}^{\prime}}(E)$ and $\operatorname{link}_{\Gamma_{F}}(E)$ are then combinatorially isomorphic for every $F \subseteq V$ that contains the carrier of $E$ (since these two links are equal if $E \cup e \notin \Gamma$ ) and the defining Equation (3-5) implies that $\ell_{V}\left(\Gamma^{\prime}, E, x\right)=\ell_{V}(\Gamma, E, x)$.

Case 2: $E \in \operatorname{link}_{\Gamma}(e)$. For $F \subseteq V$ that contains the carrier of $E$, the link $\operatorname{link}_{\Gamma_{F}^{\prime}}(E)$ is equal to either $\operatorname{link}_{\Gamma_{F}}(E)$ or to the edge subdivision of $\operatorname{link}_{\Gamma_{F}}(E)$ on $e$ in case $F$ does not or does contain the carrier of $e$, respectively. It follows from this and (3-3) that (see also [Gal 2005, Proposition 2.4.3])

$$
h\left(\operatorname{link}_{\Gamma_{F}^{\prime}}(E), x\right)= \begin{cases}h\left(\operatorname{link}_{\Gamma_{F}}(E), x\right) & \text { if } \sigma(e) \nsubseteq F, \\ h\left(\operatorname{link}_{\Gamma_{F}}(E), x\right)+x h\left(\operatorname{link}_{\Gamma_{F}}(E \cup e), x\right) & \text { if } \sigma(e) \subseteq F\end{cases}
$$

and then from (3-5) that $\ell_{V}\left(\Gamma^{\prime}, E, x\right)=\ell_{V}(\Gamma, E, x)+x \ell_{V}(\Gamma, E \cup e, x)$.

Case 3: $E \notin \Gamma$. Then we must have $E \in\{v\} * \partial(e) * \operatorname{link}_{\Gamma}(e)$ and, in particular, $v \in E$. We distinguish two subcases:

Suppose first that $E$ intersects $e$, and set $E^{\prime}=(E \backslash\{v\}) \cup e$. Then for every $F \subseteq V$ that contains the carrier of $E$ in $\Gamma^{\prime}, \operatorname{link}_{\Gamma_{F}^{\prime}}(E)=\operatorname{link}_{\Gamma_{F}}\left(E^{\prime}\right)$ (and the latter coincides with the carrier of $E^{\prime}$ in $\left.\Gamma\right)$, and hence, $\ell_{V}\left(\Gamma^{\prime}, E, x\right)=\ell_{V}\left(\Gamma, E^{\prime}, x\right)$.

Suppose finally that $E \cap e=\varnothing$, and set $E^{\prime}=(E \backslash\{v\}) \cup e$. Then for every $F \subseteq V$ that contains the carrier of $E$ in $\Gamma^{\prime}, \operatorname{link}_{\Gamma_{F}^{\prime}}(E)=\operatorname{link}_{\Gamma_{F}}\left(E^{\prime}\right) * \partial(e)$. Therefore, we have $h\left(\operatorname{link}_{\Gamma_{F}^{\prime}}(E), x\right)=(1+x) h\left(\operatorname{link}_{\Gamma_{F}}\left(E^{\prime}\right), x\right)$ for every such $F$, and hence, $\ell_{V}\left(\Gamma^{\prime}, E, x\right)=(1+x) \ell_{V}\left(\Gamma, E^{\prime}, x\right)$.

The expressions obtained for $\ell_{V}\left(\Gamma^{\prime}, E, x\right)$ and our assumption on $\Gamma$ show that, indeed, the corresponding $\gamma$-polynomial has nonnegative coefficients in all cases.

Barycentric and cluster subdivisions. As a special case of Proposition 6.1, the (first) barycentric subdivision of the simplex $2^{V}$ has nonnegative local $\gamma$-vector. Several combinatorial interpretations for its entries are given in [Athanasiadis and Savvidou 2011]. Similar results appear there for the simplicial subdivision of a simplex defined by the positive part of the cluster complex associated with a finite root system.

The following special case of Conjecture 5.4 might also be interesting to explore. The notion of a CW-regular subdivision can be defined by replacing the simplicial complex $\Delta^{\prime}$ in the definition of a topological subdivision (Definition 2.2) by a regular CW-complex; see [Stanley 1992, p. 839]. 
Question 6.2. Does Conjecture 5.4 hold for the barycentric subdivision of any CW-regular subdivision of the simplex?

\section{Acknowledgments}

The author wishes to thank an anonymous referee for helpful suggestions and Mike Davis and Volkmar Welker for useful discussions.

\section{References}

[Athanasiadis 2011] C. A. Athanasiadis, "Some combinatorial properties of flag simplicial pseudomanifolds and spheres", Ark. Mat. 49:1 (2011), 17-29. MR 2784255 Zbl 1235.52020

[Athanasiadis 2012] C. A. Athanasiadis, "Cubical subdivisions and local h-vectors", Ann. Comb. 16:3 (2012), 421-448. MR 2960014

[Athanasiadis and Savvidou 2011] C. A. Athanasiadis and C. Savvidou, "The local $h$-vector of the cluster subdivision of a simplex", Sém. Lothar. Combin. 66 (2011), Article B66c.

[Barmak 2010] J. A. Barmak, "Star clusters in independence complexes of graphs", preprint, 2010. arXiv 1007.0418

[Björner 1995] A. Björner, “Topological methods", pp. 1819-1872 in Handbook of combinatorics, vol. 2, edited by R. L. Graham et al., Elsevier, Amsterdam, 1995. MR 96m:52012 Zbl 0851.52016

[Björner et al. 1999] A. Björner, M. Las Vergnas, B. Sturmfels, N. White, and G. M. Ziegler, Oriented matroids, 2nd ed., Encyclopedia of Mathematics and its Applications 46, Cambridge University Press, Cambridge, 1999. MR 2000j:52016 Zbl 0944.52006

[Chan 1994] C. Chan, "On subdivisions of simplicial complexes: characterizing local $h$-vectors", Discrete Comput. Geom. 11:4 (1994), 465-476. MR 95b:52015 Zbl 0799.05010

[Charney and Davis 1995] R. Charney and M. Davis, "The Euler characteristic of a nonpositively curved, piecewise Euclidean manifold”, Pacific J. Math. 171:1 (1995), 117-137. MR 96k:53066 Zbl 0865.53036

[Davis and Okun 2001] M. W. Davis and B. Okun, "Vanishing theorems and conjectures for the $\ell^{2}$-homology of right-angled Coxeter groups", Geom. Topol. 5 (2001), 7-74. MR 2002e:58039 Zbl 1118.58300

[Gal 2005] Ś. R. Gal, "Real root conjecture fails for five- and higher-dimensional spheres", Discrete Comput. Geom. 34:2 (2005), 269-284. MR 2006c:52019 Zbl 1085.52005

[Munkres 1984] J. R. Munkres, Elements of algebraic topology, Addison-Wesley, Menlo Park, CA, 1984. MR 85m:55001 Zbl 0673.55001

[Postnikov et al. 2008] A. Postnikov, V. Reiner, and L. Williams, "Faces of generalized permutohedra", Doc. Math. 13 (2008), 207-273. MR 2010j:05425 Zbl 1167.05005 arXiv math/0609184

[Stanley 1992] R. P. Stanley, "Subdivisions and local h-vectors", J. Amer. Math. Soc. 5:4 (1992), 805-851. MR 93b:52012 Zbl 0768.05100

[Stanley 1996] R. P. Stanley, Combinatorics and commutative algebra, 2nd ed., Progress in Mathematics 41, Birkhäuser, Boston, 1996. MR 98h:05001 Zbl 0838.13008

[Stanley 1997] R. P. Stanley, Enumerative combinatorics, vol. 1, Cambridge Studies in Advanced Mathematics 49, Cambridge University Press, Cambridge, 1997. MR 98a:05001 Zbl 0889.05001

Received January 11, 2012. Revised May 28, 2012. 
Christos A. Athanasiadis

Division of AlgEBRA-GEOMETRY

DEPARTMENT OF MATHEMATICS

NATIONAL AND KAPODISTRIAN UNIVERSITY OF ATHENS

PANEPISTIMIOUPOLIS

15784 ATHENS

GREECE

caath@math.uoa.gr 


\title{
PACIFIC JOURNAL OF MATHEMATICS
}

\author{
http://pacificmath.org \\ Founded in 1951 by \\ E. F. Beckenbach (1906-1982) and F. Wolf (1904-1989)
}

\section{EDITORS}

V. S. Varadarajan (Managing Editor)

Department of Mathematics

University of California

Los Angeles, CA 90095-1555

pacific@math.ucla.edu

Vyjayanthi Chari

Department of Mathematics

University of California

Riverside, CA 92521-0135

chari@math.ucr.edu

\section{Robert Finn}

Department of Mathematics Stanford University

Stanford, CA 94305-2125

finn@math.stanford.edu

Kefeng Liu

Department of Mathematics

University of California

Los Angeles, CA 90095-1555

liu@math.ucla.edu
Darren Long

Department of Mathematics

University of California

Santa Barbara, CA 93106-3080

long@math.ucsb.edu

Jiang-Hua Lu

Department of Mathematics

The University of Hong Kong

Pokfulam Rd., Hong Kong jhlu@maths.hku.hk

Alexander Merkurjev

Department of Mathematics

University of California

Los Angeles, CA 90095-1555

merkurev@math.ucla.edu
Sorin Popa

Department of Mathematics University of California

Los Angeles, CA 90095-1555 popa@math.ucla.edu

Jie Qing

Department of Mathematics

University of California

Santa Cruz, CA 95064

qing@cats.ucsc.edu

Jonathan Rogawski

Department of Mathematics

University of California

Los Angeles, CA 90095-1555

jonr@math.ucla.edu

\section{PRODUCTION}

pacific@math.berkeley.edu

\section{SUPPORTING INSTITUTIONS}

ACADEMIA SINICA, TAIPEI

CALIFORNIA INST. OF TECHNOLOGY INST. DE MATEMÁTICA PURA E APLICADA KEIO UNIVERSITY

MATH. SCIENCES RESEARCH INSTITUTE NEW MEXICO STATE UNIV.

OREGON STATE UNIV.

\author{
STANFORD UNIVERSITY \\ UNIV. OF BRITISH COLUMBIA \\ UNIV. OF CALIFORNIA, BERKELEY \\ UNIV. OF CALIFORNIA, DAVIS \\ UNIV. OF CALIFORNIA, LOS ANGELES \\ UNIV. OF CALIFORNIA, RIVERSIDE \\ UNIV. OF CALIFORNIA, SAN DIEGO \\ UNIV. OF CALIF., SANTA BARBARA
}

\author{
UNIV. OF CALIF., SANTA CRUZ \\ UNIV. OF MONTANA \\ UNIV. OF OREGON \\ UNIV. OF SOUTHERN CALIFORNIA \\ UNIV. OF UTAH \\ UNIV. OF WASHINGTON \\ WASHINGTON STATE UNIVERSITY
}

These supporting institutions contribute to the cost of publication of this Journal, but they are not owners or publishers and have no responsibility for its contents or policies.

See inside back cover or pacificmath.org for submission instructions.

The subscription price for 2012 is US \$420/year for the electronic version, and \$485/year for print and electronic.

Subscriptions, requests for back issues from the last three years and changes of subscribers address should be sent to Pacific Journal of Mathematics, P.O. Box 4163, Berkeley, CA 94704-0163, U.S.A. Prior back issues are obtainable from Periodicals Service Company, 11 Main Street, Germantown, NY 12526-5635. The Pacific Journal of Mathematics is indexed by Mathematical Reviews, Zentralblatt MATH, PASCAL CNRS Index, Referativnyi Zhurnal, Current Mathematical Publications and the Science Citation Index.

The Pacific Journal of Mathematics (ISSN 0030-8730) at the University of California, c/o Department of Mathematics, 969 Evans Hall, Berkeley, CA 94720-3840, is published monthly except July and August. Periodical rate postage paid at Berkeley, CA 94704, and additional mailing offices. POSTMASTER: send address changes to Pacific Journal of Mathematics, P.O. Box 4163, Berkeley, CA 94704-0163.

PJM peer review and production are managed by EditFLOW ${ }^{\mathrm{TM}}$ from Mathematical Sciences Publishers.

PUBLISHED BY PACIFIC JOURNAL OF MATHEMATICS

at the University of California, Berkeley 94720-3840

A NON-PROFIT CORPORATION

Typeset in LATEX

Copyright $(02012$ by Pacific Journal of Mathematics 


\section{PACIFIC JOURNAL OF MATHEMATICS}

Volume $259 \quad$ No. $2 \quad$ October 2012

Flag subdivisions and $\gamma$-vectors

257

Christos A. ATHANASIAdis

Rays and souls in von Mangoldt planes

279

IGOR BELEGRADEK, ERIC CHOI and NOBUHIRO INNAMI

Isoperimetric surfaces with boundary, II

ABRAHAM FRANDSEN, DONALD SAMPSON and NEIL

STEINBURG

Cyclic branched coverings of knots and quandle homology

YUICHI KABAYA

On a class of semihereditary crossed-product orders

JOHN S. KAUTA

An explicit formula for spherical curves with constant torsion

DEMETRE KAZARAS and IVAN STERLING

Comparing seminorms on homology

JEAN-FranÇOIS LAFONT and CHRISTOPHE PITTET

Relatively maximum volume rigidity in Alexandrov geometry

NAN LI and XIAOCHUN RONG

Properness, Cauchy indivisibility and the Weil completion of a group of 421 isometries

Antonios Manoussos and Polychronis Strantzalos

Theta lifts of strongly positive discrete series: the case of $(\tilde{\mathrm{Sp}}(n), O(V)) 445$

IVAN MATIĆ

Tunnel one, fibered links

MATt RATHBUN

Fusion symmetric spaces and subfactors

HANS WENZL 